\title{
Characteristics of Dispersed ZnO-Folic Acid Conjugate in Aqueous Medium
}

\author{
Sreetama Dutta, Bichitra Nandi Ganguly ${ }^{*}$ \\ Applied Nuclear Physics Division, Saha Institute of Nuclear Physics, Kolkata, India \\ Email: "bichitra.ganguly@saha.ac.in
}

Received November 25, 2013; revised December 29, 2013; accepted January 12, 2014

\begin{abstract}
Copyright (C) 2014 Sreetama Dutta, Bichitra Nandi Ganguly. This is an open access article distributed under the Creative Commons Attribution License, which permits unrestricted use, distribution, and reproduction in any medium, provided the original work is properly cited. In accordance of the Creative Commons Attribution License all Copyrights (C) 2014 are reserved for SCIRP and the owner of the intellectual property Sreetama Dutta, Bichitra Nandi Ganguly. All Copyright (C) 2014 are guarded by law and by SCIRP as a guardian.
\end{abstract}

\begin{abstract}
The focus of this article is based on the aqueous dispersed state properties of inorganic ZnO nanoparticles (average size $\leq 4 \mathrm{~nm}$ ), their surface modification and bio-functionalization with folic acid at physiological $\mathrm{pH} \sim 7.5$, suitable for bio-imaging and targeted therapeutic application. While TEM studies of the ZnO nano-crystallites have been performed to estimate their size and morphology in dry state, the band gap properties of the freshly prepared samples, the hydrodynamic size in aqueous solution phase and the wide fluorescence range in visible region have been investigated to establish the fact that the sol is particularly suitable for bio-medical purpose in the aqueous dispersed state.
\end{abstract}

\section{KEYWORDS}

ZnO Nanoparticle; Folic Acid; Band Gap; Hydrodynamic Size; Fluorescence

\section{Introduction}

Zinc Oxide ( $\mathrm{ZnO})$ as semiconductor nano-material has wide range of multipurpose applications due to its unique optical properties, such as wide band gap $(\sim 3.37 \mathrm{eV}$ at room temperature) and high exciton binding energy (60 $\mathrm{meV}$ ) [1]. Therefore, $\mathrm{ZnO}$ nanoparticles (NPs) are important for a wide variety of optical methods owing to their size dependent properties that have been nurtured in the recent years [2]. An electronic excitation in semiconductor clusters consisting of a loosely bound electronhole pair that is generally delocalized over a NP and the band gap increases as the particle size/the cluster size of the particles diminishes (such as in case of the quantum dots, QDs). Interestingly, the trends of semiconductor colloidal systems as luminescence probes for many biological and biomedical applications have become an arena of intense research focus over the past few years [3,4]. $\mathrm{ZnO}$ as semiconductor material though environmentally friendly, with nanometer size particles of the same, may offer selective destruction of tumor cells and could be of potential use for drug delivery processes [5]

"Corresponding author. because of their cytotoxic action [6]. Such inorganic NPs need to be properly functionalized for their biological use in aqueous dispersed state, which is presently an active area of research [7] for bio-imaging and drug delivery applications [8]. In many cases, the malignancy of tumor is detected only at advanced stage when chemotherapeutic drugs become increasingly toxic to healthy cells. To improve this condition, both targeted drug delivery [9] and early detection of cancer cells continue to be extensively investigated [10]. Owing to frequent over-expression of folate receptors on the surface of malignant cells, conjugation of cytotoxic agents (could be NPs) to folic acid (FA) has been demonstrated to enhance selective drug delivery to the tumor site [11], and the detection could be achieved through fluorescence imaging or positron emission tomography (PET) techniques. It would be also pertinent to mention here that the size of the nano $\mathrm{ZnO}$ particle as QDs could find an easy entry to biological cellular objects in hydrophilic environment. In this regard, mention may be made that the surface chemistry of specially designed QDs (of ZnO NPs) readily lends them to functionalization with targeting proteins/chemi- 
cal groups, which may be a key factor to make them benign to normal cells while still retain their cancer targeting properties.

There are however, at least three primary considerations in the application of semi-conductor quantum dots (QDs) for biological applications [12]: 1) increased stability in aqueous environment over a long period of time; 2) biocompatibility and non-immunogenicity; and 3) lack of interference with the native properties of nanoparticles.

$\mathrm{ZnO}$ is an inexpensive luminescent semiconductor material compared with others [13]. Thus, the objective is to combine semiconductor nanoparticle technology with bioimaging and drug therapy. This approach is expected to have a significant impact on nano-pharmaceutical product development for cancer therapy. In particular, distribution of drug-loaded nano-carriers could be visualized in vivo [11].

In this communication, we primarily emphasize the physical study of NP [14] as QDs in aqueous phase with nearly monodispersed condition in the nascent stage, its conjugation with a biologically important molecule such as folic acid, and the transparent clear dispersion of the bioconjugated ZnO NPs in solution phase along with its optical spectroscopic absorption-emission properties. We envisage that such physical properties of $\mathrm{ZnO}$ QDs and ZnO-FA will be important for targeted drug delivery processes.

\section{Materials and Methods}

\subsection{Chemical Method}

Pure ZnO NPs were prepared by the sol-gel technique [14] from zinc acetate (A.R. grade), in alkaline solution phase using 1:1 ammonia solution (Merck, India), at $\mathrm{pH}$ 7.5. The Folic acid complexed $\mathrm{ZnO}$ (FA-ZnO) samples have been synthesized using Folic acid (M.F: $\mathrm{C}_{19} \mathrm{H}_{19} \mathrm{~N}_{7} \mathrm{O}_{6}$ procured from Sigma Life Science) in the same alkaline solution at room temperature. The requisite amounts of $\mathrm{ZnO}$ and FA-ZnO samples have been dissolved in triple distilled water (TDW) for further measurements.

\subsection{Physical Methods of Characterization of the ZnO Nanoparticles}

In order to determine physical properties of the as-prepared material, the particle size has been measured first by transmission electron microscope (TEM) and then the aqueous solvated properties/hydrodynamic size by dynamic light scattering (DLS) method after dilution with TDW as a solvent and as a function of aging. ZnO nanoparticle absorption spectra were measured using ultraviolate (UV/Vis) spectrophotometer. The light absorption properties and the emission properties have also been studied in the UV-Vis range.

The Synthesized ZnO NP samples were examined under TEM in order to study the particle morphology. In the present investigation, the particle morphology has been obtained using Tecnai S-twin, FEI made transmission electron microscope operating at $200 \mathrm{kV}$, having a resolution of $\sim 1 \AA$. For such analyses, the $\mathrm{ZnO}$ sample first has been 1) dispersed in TDW and then also in 2) iso-propanol, through a probe sonicator; a drop of the same was placed onto a carbon coated copper grid and dried at room temperature.

X-ray diffraction pattern of the nano-crystallites were measured by Seifert XDAL 3000 diffractometer with $\mathrm{CuK}_{\alpha}$ radiation (wavelength of the radiation, $\mathrm{k}=1.54 \AA$ ).

Hydrodynamic size of ZnO NPs in aqueous phase was determined by DLS. This measurement of NPs suspensions were obtained using a high performance particle size measuring instruments (Malvern Instruments Ltd., Worcestershire, U.K.), with He- Ne Laser of wavelength $633 \mathrm{~nm}$. Appropriate amounts of nano-ZnO sample were added to TDW medium. All suspensions were vigorously shaken using sonicator to break up visible clumps to yield a clear dispersion.

UV visible spectroscopy of the electronic transitions of molecules was carried out using a UV-VIS-NIR scanning spectrometer (Lamda 750, Perkin Elmer). The optical absorption spectra were measured in the range of 250 $800 \mathrm{~nm}$. From the absorption spectrum we measure the band gap of dispersed NPs in the system.

The steady-state fluorescence measurements have been made in a Spex vluoromax-3 spectrofluorimeter. A pair of $1 \mathrm{~cm} \times 1 \mathrm{~cm}$ path length quartz cuvettes were used for absorption and emission experiments of the dispersed solutions.

\section{Results and Discussion}

\subsection{Morphological Investigation by TEM}

The microstructures of pure $\mathrm{ZnO}$ and FA-ZnO NPs have been investigated by TEM under high vacuum and under dry conditions. Typical morphology of synthesized pure $\mathrm{ZnO}$ NPs has been shown in Figure 1(a), as prepared in iso-propanol (since ZnO NPs are insoluble) and they are dried on carbon coated grids. The electron diffraction pattern of the NPs in Figure 1(b). A swollen structure has been observed in case of FA-ZnO sample (shown in Figure 1(c)). Hexagonal wurtzite structure has been shown in freshly prepared pure $\mathrm{ZnO}$ sample Figure 1(d) through X-ray diffraction pattern. A histogram has been constructed (shown in Figure 2) for synthesized pure $\mathrm{ZnO}$ and FA-ZnO samples. From the histograms we can have an average estimate the particle size $\leq 4 \mathrm{~nm}$ for synthesized pure $\mathrm{ZnO}$ and $~ 11 \mathrm{~nm}$ for FA-ZnO sample. It also shows that the particle size is nearly mono-dispersed. 


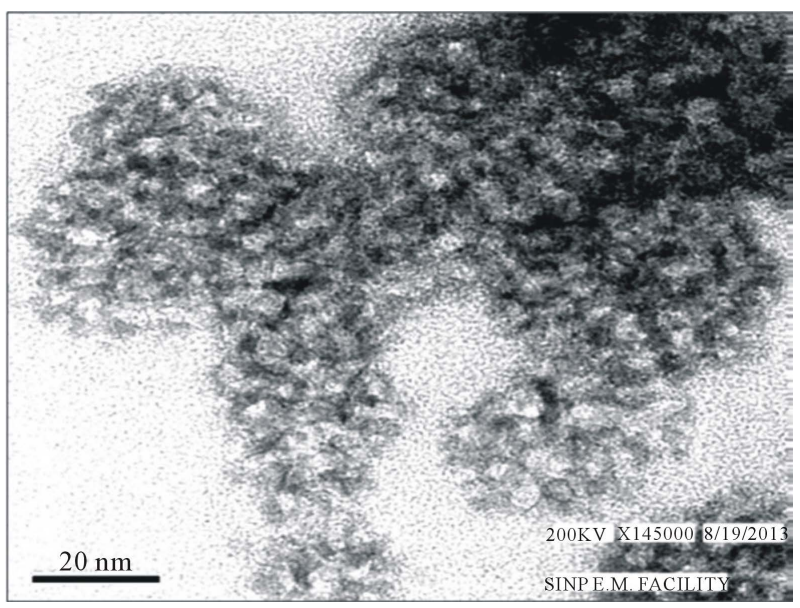

(a)

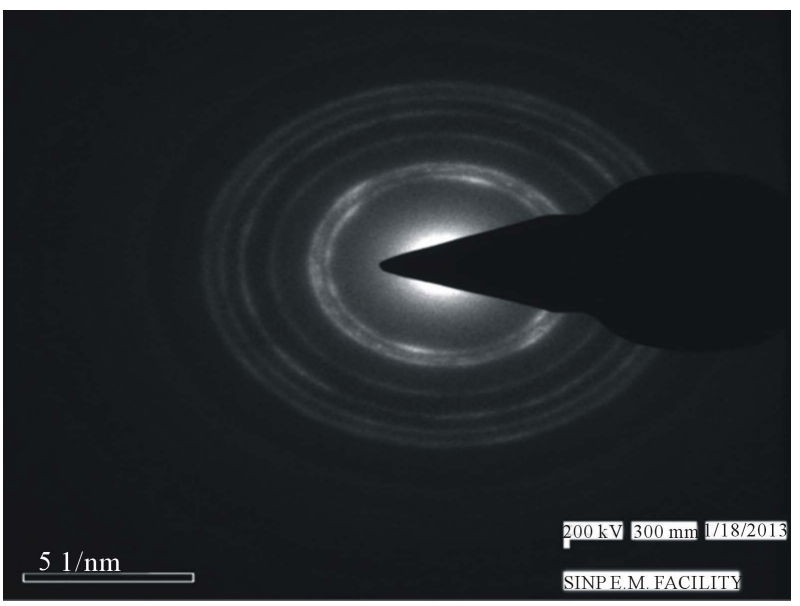

(b)

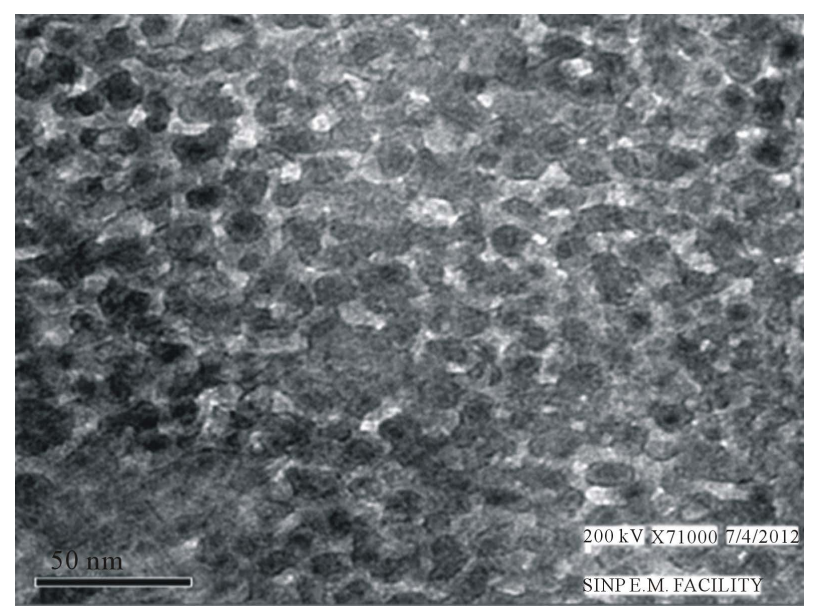

(c)

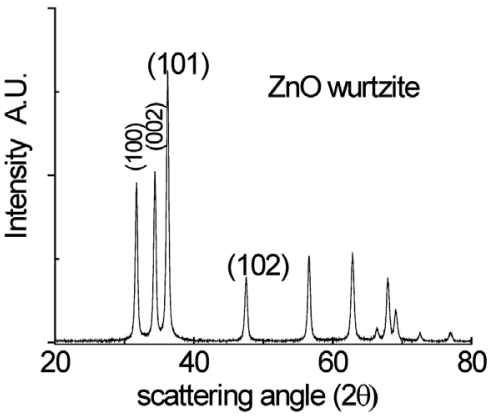

(d)

Figure 1. Transmission electron micrographs of: (a) As-prepared pure ZnO NPs, (b) Electron diffraction pattern to show the crystallinity, (c) FA-ZnO NPs and (d) X-ray diffraction pattern of ZnO.
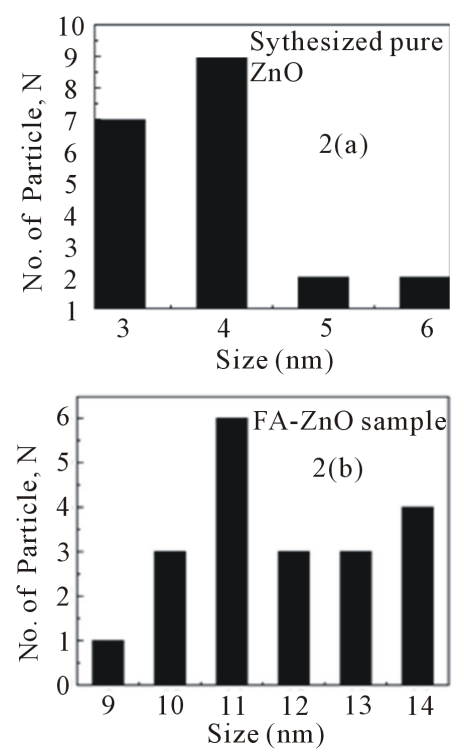

Figure 2. TEM particle size distribution histograms for synthesized (a) pure $\mathrm{ZnO}$ and (b) FA-ZnO samples.
Such small size range of NPs is useful for cancer cell selectivity [8].

\subsection{Dynamic Light Scattering (DLS) Measurement}

In solution phase, DLS results for the hydrated $\mathrm{ZnO}$ NPs explored in this study are shown in Figure 3. Dynamic light scattering method is used to measure Brownian motion of colloidal dispersed particles and to relate this to the hydrodynamic size of the particles in the dispersed solution through dynamic fluctuations of scattered light intensity. This scattered light intensity is further mathematically manipulated to relate the hydrodynamic size of the particles. An important feature of Brownian motion measured by DLS is that small particles move faster in comparison to large particles, and the relationship between the size of a particle and its speed due to Brownian motion is defined in the Stokes-Einstein equation [15]. The fundamental size distribution determined by DLS is 


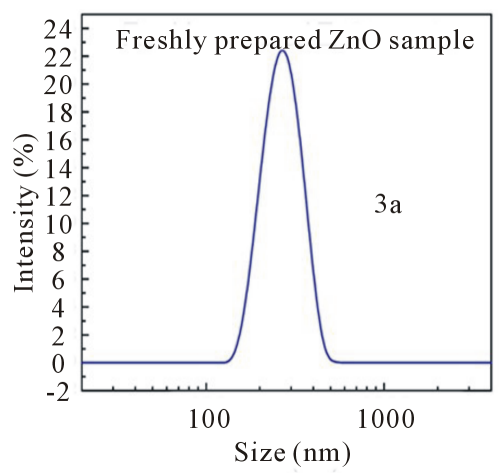

(a)

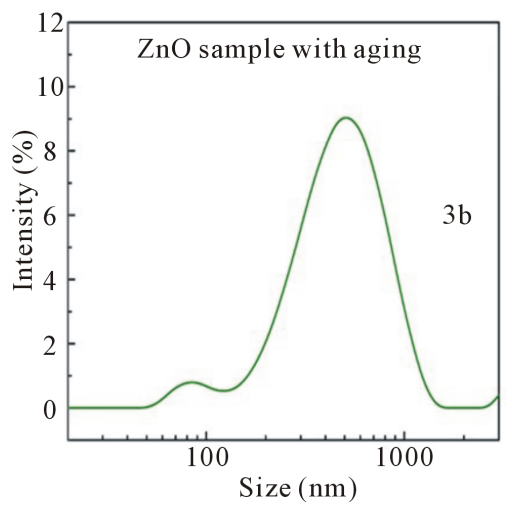

(b)

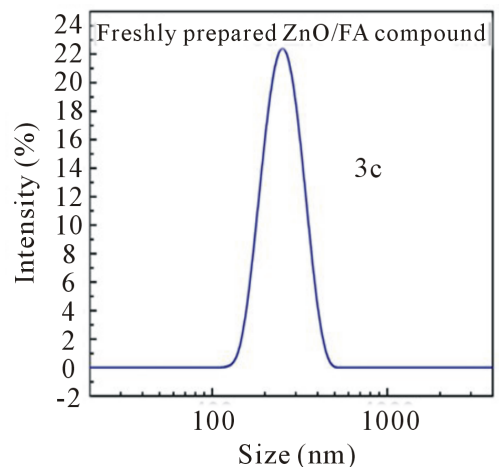

(c)

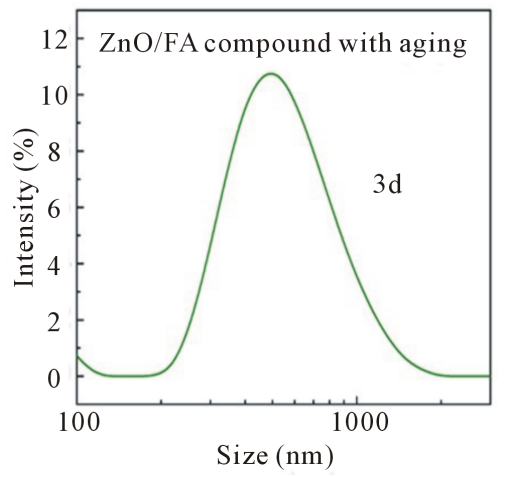

(d)

Figure 3. Particle size distribution of (a) pure $\mathrm{ZnO}$ Fresh, (b) After aging and (c) FA-ZnO Fresh, (d) After aging of NPs from DLS experiment. a scattered light intensity distribution, which can be converted to a volume distribution using Mie theory [16]. It actually measures the size of the hydrated sphere of the molecules around the $\mathrm{ZnO}$ or FA-ZnO samples, which attains stability with time. Factors that affect the diffusion speed of the NPs here are 1) ionic strength of the medium, 2) surface structure, by changing the thickness of electro-chemical double layer/Debye layer $\left(\kappa^{-1}\right)$ (Figure 4) [17]. In our method, we had dispersed the $\mathrm{ZnO}$ NPs in TDW which could produce an extended double layer of polarized water $(\mathrm{H}-\mathrm{O}-\mathrm{H})$ molecules around the particle. Also, any change due to surface conjugation of ZnO-NPs, due to large folic acid molecule affects the diffusion speed and will automatically affect the size of the NPs. The freshly prepared samples (both pure $\mathrm{ZnO}$ and FA-ZnO samples in TDW) have exhibited considerable small hydrodynamic size ( $2 \mathrm{R} \sim 388 \mathrm{~nm}$ for pure $\mathrm{ZnO}$ and $~ 518 \mathrm{~nm}$ for $\mathrm{ZnO} / \mathrm{FA}$, shown in the Table 1 ). However, the size of the NPs are very much higher than TEM nano particles, as the particles are isolated from the medium immediately after sonication and dried and put to high vacuum for investigation. In solution phase however, this discrepancy is obvious as pure $\mathrm{ZnO}$ and FAZnO NP samples further tend to get hydrated and agglomerated with time when dispersed in water [18]. It is appropriate to mention that electrostatic (charge) characteristics of $\mathrm{ZnO}$ NPs are important feature for bioapplications (it is found to be $\mathrm{pH}$ dependent [19]), in pure aqueous phase they have neutral hydrated groups attached thus dispersed $\mathrm{ZnO}$ has hydrated cell around it (shown in the Figure 4). The Debye length $\left(\kappa^{-1}\right)$, in this context refers to the mean radius of the oriented shell of water dipoles (neutral) around the NP. Since $\kappa$ is proportional to the square root of ionic strength of the medium, shell structure diminishes under ionic conditions. The solvated interaction of $\mathrm{ZnO}$ NPs in aqueous medium leaves its impact as this type of effect is persistent also from Figure 1(a), although it has been dried under vacuum.

This electrostatic interaction of NPs is physiologically important, as it drives various biological interactions. In fact, DLS results show this effect through the hydrodynamic size of the dispersed particles. In case of FAconjugated particles at physiological $\mathrm{pH} \sim 7-7.5$ of the medium, it is this electrostatic effect that drives the (negative ends) dipoles of FA molecules to orient themselves towards the $\mathrm{ZnO}$ particles through their surface positive charge. It may be mentioned that cancer cells frequently contain a high concentration of anionic phospholipids on their outer membrane and large membrane potentials [20], thus electrostatic interactions of bare ZnO NPs for such biologically driven sites deserves special mention in this context.

Ultimately, we obtain a clear transparent solution on 
Table 1. Comparison of the hydrodynamic size (2R) of synthesized pure $\mathrm{ZnO}$ and FA-ZnO samples obtained from DLS.

\begin{tabular}{cccc}
\hline Sample pure ZnO & $\begin{array}{c}\text { Size estimate } \\
\text { (TEM) }\end{array}$ & $\begin{array}{c}\text { Hydrodynamic } \\
\text { size (DLS) }\end{array}$ & $\begin{array}{c}\text { Debye } \\
\text { Layer }\end{array}$ \\
\hline Pure ZnO & nm & $388 \mathrm{~nm}$ & $192 \mathrm{~nm}$ \\
Freshly Prepared & -do- & $560 \mathrm{~nm}$ & $278 \mathrm{~nm}$ \\
After aging 1 month & -do- & $560 \mathrm{~nm}$ & $278 \mathrm{~nm}$ \\
After 2 Months & & & \\
ZnO/FA & $11 \mathrm{~nm}$ & $518 \mathrm{~nm}$ & $254 \mathrm{~nm}$ \\
Freshly prepared & -do- & $688 \mathrm{~nm}$ & $388.5 \mathrm{~nm}$ \\
After 1 month & -do- & $688 \mathrm{~nm}$ & $388.5 \mathrm{~nm}$ \\
After 2 Months & & &
\end{tabular}
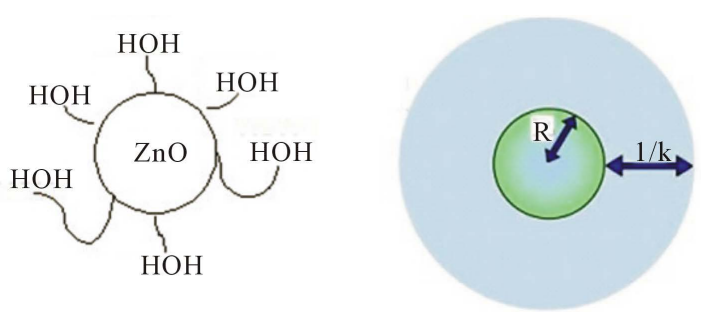

Figure 4. Schematic diagram of the hydrated layer of molecules (dipoles) arranged around the nanoparticle $\mathrm{ZnO}$ (' $\mathrm{R}$ ' denote the radius) in TDW medium showing the Debye layer $(1 / \kappa)$.

standing. Hydrodynamic sizes (2R) of both samples have been increased due to aging as is evident from Table 1. No physical change has been observed after one month time and we consider the solution phase to be stable.

\subsection{UV-Vis Spectrum Analysis}

Tailoring the band structure is a vital assignment for the research of semiconductor material, like $\mathrm{ZnO}$ and this property lies at the root of many physico-chemical processes of the cytotoxic properties of NPs. The electron and hole pair generated in these semiconductor NPs mediated processes can participate in many kinds of redox reaction cycles to generate reactive oxygen species (ROS) in cellular environments [5,21].

The band gap or band structure of the material is found to be size dependent owning to the well-known quantum confinement effects. According to Beer-Lambert's law, the absorbance $(\mathrm{A})$ is related by the equation

$$
A=\log \left(I_{0} / I\right)=\alpha d
$$

where, $I_{0}$ and $I$ are the intensities of incident and transmitted light respectively, $\alpha$ is the absorption coefficient (= $=\varepsilon$. ; where, $\varepsilon$ is the extinction coefficient and $c$ is concentration) and $d$ is the path length of absorbing solution (in this case, $d=1 \mathrm{~cm}$ and concentration is uniform).

$\mathrm{ZnO}$ is a direct band semiconductor for which $\alpha$ is related to the excitation energy by:

$$
\alpha E=A\left(E-E_{g}\right)^{1 / 2}
$$

where, $E_{\mathrm{g}}$ is a band gap energy. Standard extrapolation of absorption onset (as shown in Figure 5) [22] to $\alpha E=0$ (where $\mathrm{E}=E_{g}$ ) has been made for each samples. The band gap value of freshly prepared $\mathrm{ZnO}$ sample, shown in Figure 5, has been estimated $\sim 3.9 \mathrm{eV}$, which is quite high compared to standard sample [1,2]. S. Monticone et al. [2] has reported the band gap of freshly prepared sample as $3.75 \mathrm{eV}$. It has been also shown that the band gap value of $\mathrm{ZnO}$ NPs changes due to aging in solution [2]. Besides this, the band gap value has been changed with $\mathrm{pH}$ [23]. Seema Rani et al. [23] estimated the band gap value $\sim 3.5 \mathrm{eV}$ due to $\mathrm{pH} \sim 7$ for the particle size $~$ $7.5 \mathrm{~nm}$. In the present study, we have estimated the band gap $\sim 3.9 \mathrm{eV}$ for $\mathrm{pH} \sim 7.5$ and particle size $\sim 4 \mathrm{~nm}$ (from TEM result). From the Table 2, we observe that the band gap value has been tuned from $3.9 \mathrm{eV}$ to $2.8 \mathrm{eV}$ with increasing folic acid concentration. The band gap effect is reduced due to donor charge level since $\mathrm{ZnO}$ is ensconced by the FA [14]. According to Brus [24] the change in band gap energy for $3 d$ direct band gap material, $E_{g}$, is given as:

$$
\Delta E_{g}=\frac{\hbar^{2} \pi^{2}}{2 \mu D^{2}}-\frac{1.8 e^{2}}{\varepsilon_{2} D}
$$

where $D=2 \mathrm{R}, \mu$ is reduced mass and $\varepsilon_{2}$ is dielectric constant.

The quantum confinement is material specific because it depends on dielectric constant of the material. The analysis seems to work particularly well for $\mathrm{ZnO}$ particles when the size gets smaller than $5-6 \mathrm{~nm}$. In this case, the NPs average size obtained is $\sim 4 \mathrm{~nm}$.

Therefore, quantum confinement effect is observed and we find a considerable increase of band gap energy. But the band gap effect decreases due to FA ensconced charge at donor level, see Figure 6 and also (Schematic diagram shown) in Figure 7 with the surface modification of the NPs.

\subsection{Analysis of Emission Spectrum}

Photo activation of ZnO NPs has been effectively utilized in generating greater levels of ROS release which if effectively targeted to cancer cells will lead to selective destruction $[5,20]$. Recent reports reveal that illumination or photo-excitation of $\mathrm{ZnO}$ conjugated $\mathrm{ZnO}$ NPs resulted in synergistic cytotoxic action in ovarian cancer cells, where as little cytotoxicity has been observed under dark condition [24].

We found synthesized pure $\mathrm{ZnO}$ and FA-ZnO samples dispersed in aqueous medium show a clear emission spectrum in the region $400-500 \mathrm{~nm}$ in Figure 8. Folic acid (concentration) ensconcement can help to overall 


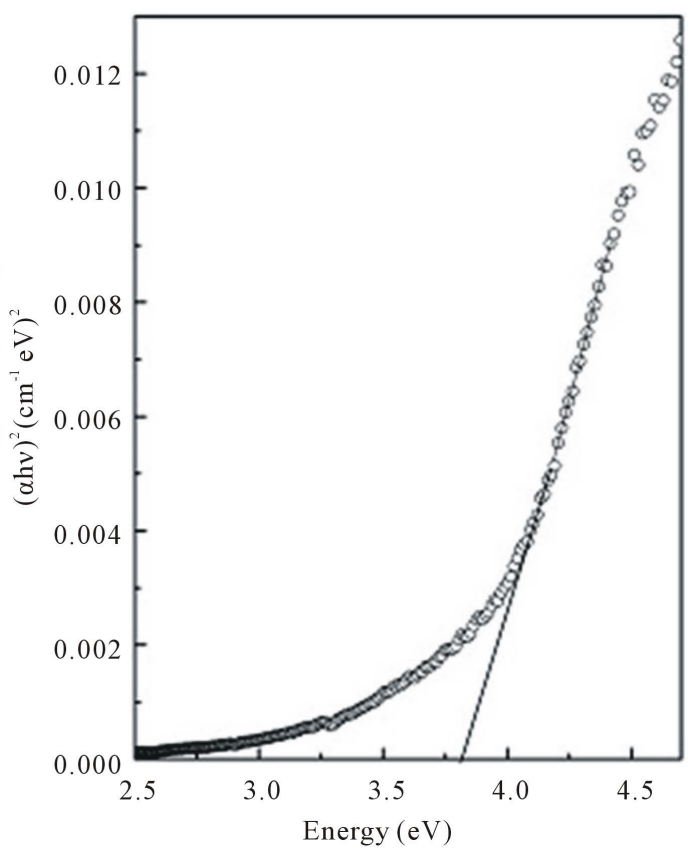

Figure 5. A standard plot of $(\alpha h v)^{2}$ versus energy for synthesized pure ZnO-NPs, showing the extrapolation to abscissa for band gap measurement.
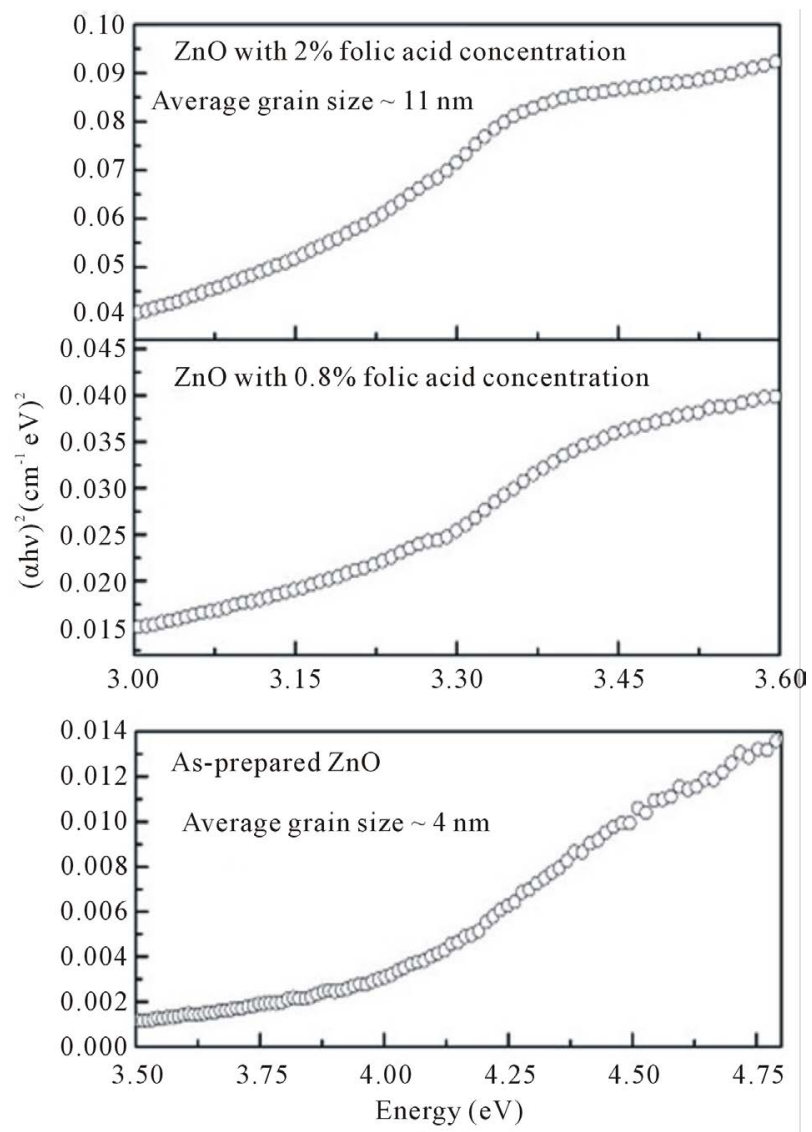

Figure 6. $(\alpha h v)^{2}$ versus energy plot of synthesized pure $\mathrm{ZnO}$ NPs and FA-ZnO NPs (at different concentration of FA) samples with different particle size.

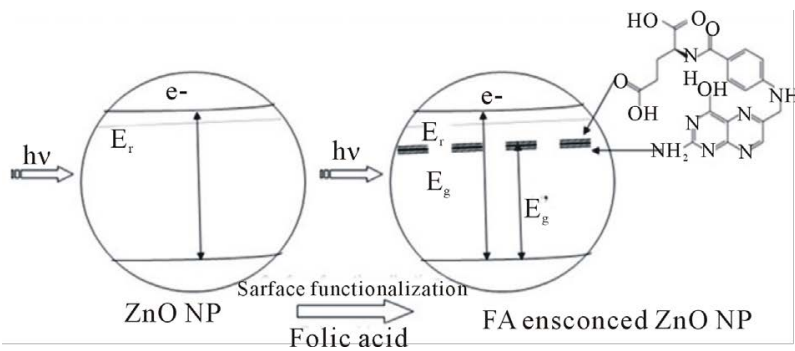

Figure 7. Schematic diagram of FA ensconced charge donor level decreasing the transition level.

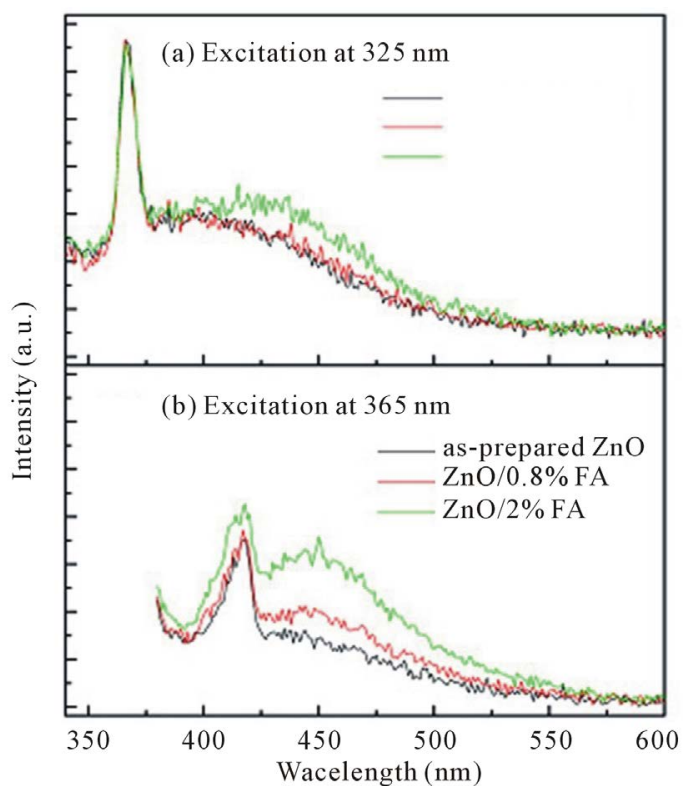

Figure 8. Fluorescence spectra of synthesized pure $\mathrm{ZnO}$ and FA-ZnO samples at different excitation wavelength.

Table 2. Comparison of the hydrodynamic size of synthesized pure ZnO and FA-ZnO samples obtained from DLS.

\begin{tabular}{|c|c|c|}
\hline $\begin{array}{l}\text { Particle size } \\
\text { ZnO NPs }\end{array}$ & Method & $\begin{array}{c}\text { Band } \\
\text { gap }\end{array}$ Reference \\
\hline $41 \mathrm{~nm}$ & Sol-gel method, pure $\mathrm{ZnO}$ & $3.3 \mathrm{eV}$ \\
\hline $20 \mathrm{~nm}$ & Sol-gel method, with FA & $3.22 \mathrm{eV}$ \\
\hline- & Freshly prepared colloidal ZnO NPs & $3.75 \mathrm{eV}$ \\
\hline $7.5 \mathrm{~nm}$ & Sol-gel method, $\mathrm{pH} \sim 7$ & $3.5 \mathrm{eV}$ \\
\hline $4 \mathrm{~nm}$ & Aqueous medium, $\mathrm{pH} \sim 7.5$, pure $\mathrm{ZnO}$ & $3.9 \mathrm{eV}$ This work \\
\hline $11 \mathrm{~nm}$ & Aqueous medium, $\mathrm{Ph} \sim 7.5$, FA-ZnO & $2.8 \mathrm{eV}$ \\
\hline
\end{tabular}

enhancement of the intensity of florescence emission [25], the emission peak $\lambda_{\max } \sim 450 \mathrm{~nm}$ (slightly red shifted in both the cases than pure $\mathrm{ZnO}$ ). In case of excitation energy at $365 \mathrm{~nm}$, in FA-ZnO samples the emission is increased by two times, which means $\mathrm{ZnO}$ in organic confinement and FA templated structure gives better fluorescence as shown from the Figure 8(b). This property can be utilized in cytotoxic action to generateROS cascade in cellular environment.

Finally, it needs to be mentioned: we find a Raman 
scattering peak at $367 \mathrm{~nm}$, for excitation energy $\lambda_{\mathrm{ex}} \sim 325$ $\mathrm{nm}$ and $417 \mathrm{~nm}$, for $\lambda_{\mathrm{ex}} \sim 365 \mathrm{~nm}$, which is due to $\mathrm{ZnO}$ particles being dipolar and Raman active and can be also useful for medicine [26]

\section{Conclusion}

Bio-conjugation of metal oxide nanoparticle is an important pathway to novel imaging probes and targeting agents, and through our finding, it is clear that FA functionalized $\mathrm{ZnO}$ is a better aqueous dispersed and stable system than $\mathrm{ZnO}$ itself, which is evident from its hydrodynamic radius. The system FA-ZnO in aqueous phase shows fluorescence in the visible region that can have potential application probability for targeting biologically active tumor cells which have folate receptor proteins. It can serve to be used as fluorescent probe in biological staining and diagnostics because the substance is a photochemically stable system.

\section{Acknowledgements}

The authors acknowledge the technical help received for measurements from Biophysics Division, SINP (Mr. Pulak Ray) for TEM, Surface Physics Division, SINP (Ms Tanusree Samanta) for DLS and Division of Chemical Sciences (Mr. Ajoy Das) for Fluorescence measurements. The authors also thank for the measurements of UV-Vis spectroscopy and other technical help to Soma Roy.

\section{REFERENCES}

[1] S. Dutta, S. Chattopadhyay, A. Sarkar, M. Chakrabarti, D. Sanyal and D. Jana, "Role of Defects in Tailoring Structural, Electrical and Optical Properties of ZnO,” Progress in Materials Science, Vol. 54, No. 1, 2009, pp. 89-136. http://dx.doi.org/10.1016/j.pmatsci.2008.07.002

[2] S. Monticone, R. Tufeu and A. V. Kanaev, "Complex Nature of the UV and Visible Fluorescence of Colloidal ZnO Nanoparticles,” The Journal of Physical Chemistry B, Vol. 102, No. 16, 1998, pp. 2854-2862. http://dx.doi.org/10.1021/jp973425p

[3] M. J. Murcia and C. A. Naumann, "Biofunctionalization of Flu-Orescent Nanoparticles,” In: S. S. Challa and R. Kumar, Eds., Nanotechnologies for the Life Sciences, Vol. 1. Biofunctionalization of Nanomate Rials, WILEY-VCH Verlag GmbH \& Co. KGaA, Weinheim, 2005, pp. 1-40.

[4] N. Erathodiyil and J. Y. Ying, "Functionalization of Inorganic Nanoparticles for Bioimaging Applications," Accounts of Chemical Research, Vol. 44, No. 10, 2011, pp. 925-935. http://dx.doi.org/10.1021/ar2000327

[5] J. W. Rasmussen, E. Martinez, P. Louka and D. G. Wingett, "Zinc Oxide Nanoparticles for Selective Destruction of Tumor Cells and Potential for Drug Delivery Applications," Expert Opinion on Drug Delivery, Vol. 7, No. 9, 2010, pp. 1063-1077.

http://dx.doi.org/10.1517/17425247.2010.502560

[6] C. Hanley, J. Layne, A. Punnoose, K. M. Reddy, I.
Coombs, A. Coombs, K. Feris and D. Wingett, "Preferential Killing of Cancer Cells and Activated Human T Cells Using ZnO Nanoparticles,” Nanotechnology, Vol. 19, No. 29, 2008, pp. 295103-295113.

http://dx.doi.org/10.1088/0957-4484/19/29/295103

[7] S. T. Selvan, T. T. Y. Tan, D. K. Yi and N. R. Jana, "Functional and Multifunctional Nanoparticles for Bioimaging and Biosensing," Langmiur, Vol. 26, No. 14, 2010, pp. 11631-11641.

http://dx.doi.org/10.1021/la903512m

[8] C. Hanley, A. Thurber, C. Hanna, et al., "The Influences of Cell Type and ZnO Nanoparticle Size and Immune Cell Cytotoxicity and Cytokine Induction,” Nanoscale Research Letters, Vol. 4, No. 12, 2009, pp. 1409-1420. http://dx.doi.org/10.1007/s11671-009-9413-8

[9] P. Ruenraroengsak, J. M. Cook and A. T. Florence, “Nanosystem Drug Targeting: Facing up to Complex Realities," Journal of Controlled Release, Vol. 141, No. 3, 2010, pp. 265-276. http://dx.doi.org/10.1016/j.jconrel.2009.10.032

[10] S. M. Moghimi and A. R. Rajabi-Siahboomi, "Recent Advances in Cellular, Sub-Cellular and Molecular Targeting," Advanced Drug Delivery Reviews, Vol. 41, No. 2, 2000, pp. 129-133. http://dx.doi.org/10.1016/S0169-409X(99)00060-5

[11] A. Guaragna, A. Chiaviello, C. Paolella, D. D’Alonzo and G. Palumbo, "Synthesis and Evaluation of Folate-Based Chlorambucil Delivery Systems for Tumor-Targeted Chemotherapy," Bioconjugate Chemistry, Vol. 23, No. 1, 2012, pp. 84-96. http://dx.doi.org/10.1021/bc200410d

[12] Y. Wang and L. Chen, "Quantum Dots, Lighting up the Research and Development of Nanomedicine," Nanomedicine: Nanotechnology, Biology, and Medicine, Vol. 7, No. 4, 2011, pp. 385-402. http://dx.doi.org/10.1016/j.nano.2010.12.006

[13] Y. L. Liu, Y. H. Yang, H. F. Yang, Z. M. Liu, G. L. Shen and R. Q. Yu, "Nanosized Flower-Like ZnO Synthesized by a Simple Hydrothermal Method and Applied as Matrix for Horseradish Peroxidase Immobilization for ElectroBiosensing,” Journal of Inorganic Biochemistry, Vol. 99, No. 10, 2005, pp. 2046-2053. http://dx.doi.org/10.1016/j.jinorgbio.2005.07.001

[14] S. Dutta and B. N. Ganguly, "Characterization of ZnO Nanoparticles Grown in Presence of Folic Acid Template," Journal of Nanobiotechnology, Vol, 10, No. 1, 2012, pp. 29-38. http://dx.doi.org/10.1186/1477-3155-10-29

[15] A. L. Kholodenko and J. F. Douglas, "Generalized StokesEinstein Equation for Spherical Particle Suspensions," Physical Review E, Vol. 51, No. 2, 1995, pp. 1081-1090. http://dx.doi.org/10.1103/PhysRevE.51.1081

[16] M. Kerker, "The Scattering of Light and the Electromagnetic Radiation,” Academic Press, New York, 1969.

[17] G. W. Castellan, "Physical Chemistry,” 3rd Edition, Narosa, New Delhi, 2002, p. 364.

[18] R. C. Murdock, L. Braydich-Stolle, A. M. Schrand, J. J. Schlager and S. M. Hussain, "Characterization of Nanomaterial Dispersion in Solution Prior to in Vitro Exposure Using Dynamic Light Scattering Technique,” Toxicological Sciences, Vol. 101, No. 2, 2008, pp. 239-253. 
http://dx.doi.org/10.1093/toxsci/kfm240

[19] M. Nagao, "Physiosorption of Water on Zinc Oxide Surface,” The Journal of Physical Chemistry, Vol. 75, No. 25, 1971, pp. 3822-3828.

http://dx.doi.org/10.1021/j100694a007

[20] M. Abercrombie and E. J. Ambrose, "The Surface Properties of Cancer Cells: A Review,” Cancer Research, Vol. 22, 1962, pp. 525-548.

[21] S. W. Ryter, H. P. Kim, A. Hoetzel, et al., "Mechanisms of Cell Death in Oxidative Stress," Antioxid Redox Signal, Vol. 9, No. 1, 2007, pp. 49-89. http://dx.doi.org/10.1089/ars.2007.9.49

[22] J. Pancove, “Optical Processes in Semiconductors,” Prentice-Hall, Upper Saddle River, 1979.

[23] S. Rani, P. Suri, P. K. Shishodia and R. M. Mehra, "Synthesis of Nanocrystalline ZnO Powder via Sol-Gel Route for Dye-Sensitized Solar Cells," Solar Energy Materials \& Solar Cells, Vol. 92, No. 12, 2008, pp. 1639-1645. http://dx.doi.org/10.1016/j.solmat.2008.07.015
[24] L. Brus, "Electronic Wave Functions in Semiconductor Clusters: Experiment and Theory," The Journal of Physical Chemistry, Vol. 90, No. 12, 1986, pp. 2555-2560. http://dx.doi.org/10.1021/j100403a003

[25] J. R. Lakowicz, "Principles of Fluorescence Spectroscopy,” Plenum Press, New York, 1983. http://dx.doi.org/10.1007/978-1-4615-7658-7

[26] J. Minbiao, et al., "Rapid, Label-Free Detection of Brain Tumors with Stimulated Raman Scattering Micro Scopy," Science Translational Medicine, Vol. 5, No. 201, 2013, p. 201ra119. 